\title{
Cluster Analysis Assisted Float-Encoded Genetic Algorithm for a More Automated Characterization of Hydrocarbon Reservoirs
}

\author{
Norbert Péter Szabó ${ }^{1}$, Mihály Dobróka ${ }^{1}$, Réka Kavanda ${ }^{2}$ \\ ${ }^{1}$ Department of Geophysics, University of Miskolc, Miskolc, Hungary \\ ${ }^{2}$ DACHS GmbH., Miskolc, Hungary \\ Email: norbert.szabo.phd@gmail.com,dobroka@uni-miskolc.hu,kavreka@gmail.com
}

Received August 25, 2013; revised September 25, 2013; accepted October 2, 2013

Copyright (C) 2013 Norbert Péter Szabó et al. This is an open access article distributed under the Creative Commons Attribution License, which permits unrestricted use, distribution, and reproduction in any medium, provided the original work is properly cited.

\begin{abstract}
A genetic algorithm-based joint inversion method is presented for evaluating hydrocarbon-bearing geological formations. Conventional inversion procedures routinely used in the oil industry perform the inversion processing of borehole geophysical data locally. As having barely more types of data than unknowns in a depth, a set of marginally over-determined inverse problems has to be solved along a borehole, which is a rather noise sensitive procedure. For the reduction of noise effect, the amount of overdetermination must be increased. To fulfill this requirement, we suggest the use of our interval inversion method, which inverts simultaneously all data from a greater depth interval to estimate petrophysical parameters of reservoirs to the same interval. A series expansion based discretization scheme ensures much more data against unknowns that significantly reduces the estimation error of model parameters. The knowledge of reservoir boundaries is also required for reserve calculation. Well logs contain information about layer-thicknesses, but they cannot be extracted by the local inversion approach. We showed earlier that the depth coordinates of layerboundaries can be determined within the interval inversion procedure. The weakness of method is that the output of inversion is highly influenced by arbitrary assumptions made for layer-thicknesses when creating a starting model (i.e. number of layers, search domain of thicknesses). In this study, we apply an automated procedure for the determination of rock interfaces. We perform multidimensional hierarchical cluster analysis on well-logging data before inversion that separates the measuring points of different layers on a lithological basis. As a result, the vertical distribution of clusters furnishes the coordinates of layer-boundaries, which are then used as initial model parameters for the interval inversion procedure. The improved inversion method gives a fast, automatic and objective estimation to layer-boundaries and petrophysical parameters, which is demonstrated by a hydrocarbon field example.
\end{abstract}

Keywords: Hierarchical Cluster Analysis; Genetic Algorithm; Well-Logging; Interval Inversion

\section{Introduction}

Borehole geophysical measurements are extensively used in hydrocarbon exploration for collecting high resolution in-situ information about subsurface geological formations. Well-logging data measured by different physical principles are recorded along depth in the form of well logs [1]. The primary aim of the interpretation of observations is the lithological separation of the succession of strata and the estimation of thicknesses and petrophysical properties of formations (such as porosity, water saturation, mineral content, permeability, etc.) for the calculation of oil and gas reserves. The most advanced tools of well $\log$ analysis are based on geophysical inversion methods. By assuming a petrophysical model, one can calculate theoretical well logs, which are compared to real ones measured in the borehole. The initial model is progressively refined in an iteration procedure until a proper fit is achieved between the predictions and observations. The estimated model obtained in the last iteration step is accepted as the solution of the inverse problem that represents the most probable geological structure. The mathematical basis and computer implementation of traditional well-logging inversion methods, which solve the inverse problem depth by depth separately, can be found in [2-4]. The positions of layer-boundaries cannot be extracted from the local data set by this inver- 
sion method. Instead they are picked out on the lithological logs manually. A novel inversion method that inverts a data set of a greater depth interval to estimate the variation of petrophysical parameters and thicknesses along a borehole was developed by the Department of Geophysics, University of Miskolc. The inversion methodology named interval inversion has been applied to solve several hydrocarbon exploration problems [5-9].

Multivariate statistical methods are proven to be powerful tools in formation evaluation [10]. Regression, factor and cluster analysis are widely used to find correlations between petrophysical variables, to reduce problem dimensionality or explore non-measurable background variables as well as to separate observed data into distinct groups of different lithological characters. The principles of clustering techniques are detailed in [11], to which a variety of petrophysical applications can be found in the literature, e.g. in [12-15]. In this study, we apply the hierarchical form of cluster analysis to separate nearly homogeneous beds of shaly-sand sequences on a lithological basis. This technique is able to specify the coordinates of layer boundaries in an automatic procedure, which are then used as input for solving a global optimization-based interval inversion procedure. The workflow enables a fast and automatic estimation of layer-boundaries and petrophysical parameters to provide the log analysts and reservoir engineers with accurate and reliable information for planning the exploitation of hydrocarbon fields.

\section{Theory of Interpretation Method}

\subsection{Well-Logging and Inversion}

Several books deal with the basics of well-logging surveying and interpretation methods, among them many was written chiefly for petroleum geologists $[16,17]$. The classification of well logs can be made by parameter sensitivities [18], which in this particular case inform how the data are influenced by the petrophysical parameters, separately. Accordingly, three main groups of measurements can be distinguished, i.e. lithology, porosity and saturation sensitive logs.

In hydrocarbon exploration relatively high cost measurements are made to increase the probability of finding an economically valuable oil/gas field. Normally, a field data set consists of natural gamma-ray intensity $(G R)$, spectral gamma-ray intensity such as potassium $(K)$, thorium $(T H)$, uranium $(U)$, and spontaneous potential $(S P)$ data (lithology logs). Porosity logs comprise formation density $(D E N)$, neutron porosity $(N P H I)$ and acoustic traveltime $(A T)$ data. Resistivity tools measure the formation resistivity with different penetration depth from the borehole wall. In this study, true resistivity $(R T) \log$ is applied that represents the corrected resistivity of the part of formation not invaded by the drilling mud (saturation $\log$ ). The observed data are inverted to derive petrophysical properties of formations such as effective porosity, water saturation at different penetration depth, mineral volumes, shale content and permeability that cannot be measured directly, but necessary for the calculation of hydrocarbon reserves. Since the measured data can be related to petrophysical parameters by probe response equations [5], thus the latter can be estimated by an iterative inversion procedure. The principles of geophysical inversion and related issues are detailed in [19]. Inversion methods are preferred as they use all suitable $\operatorname{logs}$ instead of one or two logs to reduce the estimation error of petrophysical parameters. To further increase the performance of inverse modeling as much good quality prior information as possible should be given and proper optimization strategy must be posed when they are applied.

\subsection{Local Inverse Modeling}

Let $\boldsymbol{m}$ be the column vector of model parameters such as porosity $(P O R)$, water saturation in the flushed zone invaded by mud filtrate $(S X 0)$, water saturation of undisturbed formation $(S W)$, shale volume $(V S H)$, quartz content $(V S D)$ in a given depth. Well-logging data types measured at the same measuring point are also represented in a column vector $\boldsymbol{d}^{(m)}$. If we consider the data set listed in Section 2.1, an inverse problem with 5 unknowns and 9 data has to be solved in each depth. In this case, the overdetermination (data-to-unknowns) ratio is 1.8 .

Theoretical (calculated) well-logging data are connected to the petrophysical model nonlinearly as $\boldsymbol{d}^{(c)}=$ $\boldsymbol{g}(\boldsymbol{m})$, where $\boldsymbol{g}$ represents a set of probe response functions. Substituting the initial guess of model parameters to the empirically derived response equations one can calculate a local data set in the measuring point. The solution of the inverse problem is found at the minimal misfit between the observed and predicted data [19]. The Euclidean norm of the deviation between measured and calculated data vectors is applied as an objective function for the optimization

$$
E=\sum_{k=1}^{N}\left(\frac{d_{k}^{(m)}-d_{k}^{(c)}}{\sigma_{k}}\right)^{2}=\min
$$

where $\sigma_{k}$ is the variance of the $k$-th well log depending on the probe type and borehole conditions ( $N$ is the number of applied probes). To optimize Equation (1), the Weighted Least Squares method (WLSQ) is used, where the actual model is gradually refined by $\boldsymbol{m}=\boldsymbol{m}^{(0)}+\boldsymbol{\delta m}$, where $\boldsymbol{m}^{(0)}$ is the initial model and $\boldsymbol{\delta} \boldsymbol{m}$ is the model correction vector. By introducing the diagonal weighting matrix $W_{k k}=1 / \sigma_{k}^{2}(k=1,2, \ldots N)$ including data variances, the 
vector of model corrections in a given iteration step can be estimated by

$$
\boldsymbol{\delta} \boldsymbol{m}=\left(\boldsymbol{G}^{\mathrm{T}} \boldsymbol{W} \boldsymbol{G}\right)^{-1} \boldsymbol{G}^{\mathrm{T}} \boldsymbol{W} \boldsymbol{\delta} \boldsymbol{d}
$$

where $\boldsymbol{G}$ denotes the Jacobi's matrix containing partial derivatives of data with respect to model parameters and $\delta \boldsymbol{d}$ is the difference between the measured and calculated data vector ( $\mathrm{T}$ is the symbol of matrix transpose). The quality check of inversion results is based on the following connection between the uncertainties of observed data and model parameters [19]

$$
\operatorname{cov} \boldsymbol{m}=\boldsymbol{M} \operatorname{cov} \boldsymbol{d} \boldsymbol{M}^{\mathrm{T}}
$$

where covm and covd denote the data and model covariance matrices, respectively, and $\boldsymbol{M}$ is the generalized inverse matrix of the actual inversion method. The latter can be expressed with the proper combination of matrix $\boldsymbol{G}$. If we know data variances from covd, Equation (3) gives the estimation errors of model parameters at the end of the inversion procedure. They are obtained from the main diagonal of model covariance matrix as $\sigma_{m, I}=$ $\operatorname{sqrt}\left(\operatorname{diag}\left(\operatorname{covm} m_{i i}\right)\right)$. For measuring the distance between the observed and calculated data the RMS error is normally used.

\subsection{Genetic Algorithm-Based Interval Inversion}

Linearized optimization methods work properly and quickly only when an initial model sufficiently close to the solution is available. However, in cases when poor prior information or rather noisy data is provided the WLSQ procedure can be easily trapped in a local minimum of Equation (1). To avoid localities a global optimization method such as Genetic Algorithm (GA) is used that searches the absolute extreme of the objective function. GA belongs to the class of evolutionary algorithms that solves optimization problems using the analogy of natural selection of living populations [20]. Nowadays the most preferred variant is the Float-Encoded GA that improves a model population represented by model parameters from the domain of real numbers in an iteration procedure [21].

In the population each individual has a fitness value representing its survival capability. During the genetic process the fittest individuals reproduce more successfully in the subsequent generations than those who have relatively low fitness. To achieve the best solution, the fitness function is maximized by using genetic operations in a random optimum-seeking procedure. From the point of view of well-logging inverse problem a petrophysical model has large fitness when the misfit is relatively small between the observed and calculated data. In well-logging inversion normally $30-100$ model take part in the selection process. To reach the absolute maximum of fitness function a proper combination of genetic operators such as selection, crossover, mutation and reproduction is used (GA search in Figure 1). According to our experience after some tens of thousands generations (iteration steps) the fittest individual of the last generation can be accepted as the optimal petrophysical model. More details of the GA-based inversion procedure can be found in $[5,9]$.

Local inversion methods (Chapter 2.2.) process barely more data than unknowns, where the accuracy of solution highly depends on the noise level of data and the initial guess of the petrophysical model. To increase the overdetermination of the inverse problem, we define a set of probe response functions as $\boldsymbol{d}(z)=\boldsymbol{g}(\boldsymbol{m}(z))$, which is valid in a greater depth interval. In the response functions the data and model parameters are varying with depth. To discretize the depth variations of petrophysical unknowns we suggest a series expansion technique

$$
m_{i}(z)=\sum_{q=1}^{Q_{i}} B_{q}^{(i)} \Psi_{q}(z)
$$

where $m_{i}$ denotes the $i$-th model parameter, $B_{q}$ is the $q$-th expansion coefficient, $\Psi_{q}$ is the $q$-th basis function, $Q_{i}$ is the requisite number of coefficients describing the $i$-th unknown. Basis functions in Equation (4) are known and arbitrarily chosen. For instance, in homogeneous layers a combination of heaviside functions $(u)$ is advantageous to use as basis function. From one hand, using the basis function $Y_{q}(z)=u\left(z-Z_{q-1}\right)-u(z-Z q)$, each petrophysical parameter in the $q$-th layer (where $\Psi_{q}(z)=1$ ) can be described by one series expansion coefficient. On the other hand, we can introduce $Z_{q-1}, Z_{q}$ upper and lower depth coordinates of the $q$-th layer as unknown in the inverse problem [5]. For describing inhomogeneous intervals polynomial basis functions can be used [7]. The model parameter vector to be determined by inversion is $\boldsymbol{m}=[\boldsymbol{B}, \boldsymbol{Z}]^{\mathrm{T}}$, where $\boldsymbol{B}$ and $\boldsymbol{Z}$ vectors contain all series expansion coefficients given in Equation (4) and layer-

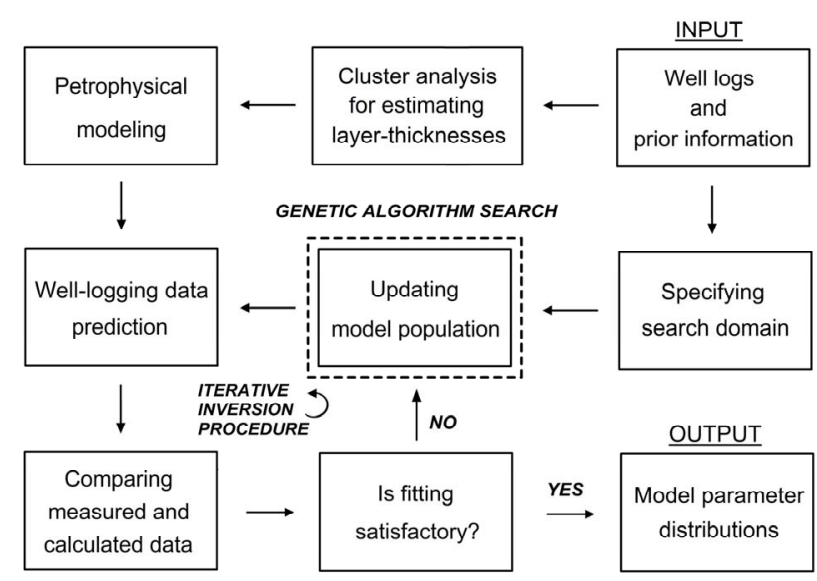

Figure 1. Workflow of the GA-based inversion procedure. 
boundary coordinates (or thicknesses) in the processed interval, respectively.

The fitness function of the GA process is inversely connected to the objective function of the well-logging inverse problem. We tested two types of fitness functions. The first one follows the idea of traditional inversion methods represented by the objective function in Equation (1)

$$
F_{1}(\boldsymbol{m})=-\sum_{p=1}^{P} \sum_{k=1}^{N}\left(\frac{d_{p k}^{(m)}-d_{p k}^{(c)}}{\sigma_{p k}}\right)^{2}=\max ,
$$

where $P$ is the total number of measuring points. The minimization of the weighted least squares criterion in Equation (5) leads to optimal solution as well-logging data have different magnitudes and measurement units. The only weakness of weighting by variances is that we have to know standard deviations of all data types in each depth. In fact, the variances of data in most of the cases are not known (just from literature given for probe types), because normally we measure only once in a depth point. Our experience shows that an optimal solution can be given by normalizing the individual data differences by the measured data

$$
F_{2}(\boldsymbol{m})=-\sum_{p=1}^{P} \sum_{k=1}^{N}\left(\frac{d_{p k}^{(m)}-d_{p k}^{(c)}}{d_{p k}^{(m)}}\right)^{2}=\max .
$$

To compare the performance of the $F_{1}$ and $F_{2}$ based interval inversion procedures a four-layered petrophysical model was defined (Table 1). We calculated synthetic data by the exact model parameters (POR, $S X 0, S W$, $V S H, V S D)$. To synthetic well logs $5 \%$ Gaussian distributed noise was added to produce quasi measured data

Table 1. GA interval inversion results for synthetic case.

\begin{tabular}{cccccc}
\hline \multirow{2}{*}{ Layer } & \multicolumn{5}{c}{ Petrophysical parameters as inversion unknowns } \\
\cline { 2 - 6 } & POR & SXO & SW & VSH & VSD \\
\hline \multirow{3}{*}{1} & $0.19^{*}$ & 0.79 & 0.39 & 0.30 & 0.49 \\
& $0.20^{* *}$ & 0.79 & 0.40 & 0.30 & 0.49 \\
& $0.20^{* * *}$ & 0.80 & 0.40 & 0.30 & 0.50 \\
\multirow{2}{*}{2} & 0.09 & 1.01 & 1.01 & 0.79 & 0.11 \\
& 0.10 & 1.00 & 1.01 & 0.80 & 0.11 \\
& 0.10 & 1.00 & 1.00 & 0.80 & 0.10 \\
3 & 0.29 & 0.81 & 0.30 & 0.11 & 0.60 \\
& 0.30 & 0.80 & 0.30 & 0.10 & 0.60 \\
& 0.30 & 0.80 & 0.30 & 0.10 & 0.60 \\
4 & 0.10 & 0.99 & 0.98 & 0.60 & 0.29 \\
& 0.10 & 0.99 & 0.98 & 0.60 & 0.30 \\
& 0.10 & 1.00 & 1.00 & 0.60 & 0.30 \\
\hline
\end{tabular}

* Maximization of fitness function weighted by data variances $\left(F_{1}\right) .{ }^{* *}$ Maximization of fitness function weighted by observed data $\left(F_{2}\right)$. ${ }^{* * *}$ Exactly known (target) model parameter. substituting real measurements. The input of the inversion procedure was a noisy well-logging data set includeing 1400 data $(20 \mathrm{~m}$ processed length, $0.1 \mathrm{~m}$ sampling interval, 7 types of well logs).

Series expansion was developed for describing a layerwise homogeneous model. There were 20 unknowns against data, thus the overdetermination ratio (70) was almost 40 times higher than that of local inversion (Section 2.2.). In Equation (5) the standard deviations of data were calculated empirically for each data types layer by layer. For the characterization of misfit we used the relative data and model distances. The first measures the difference between the measured and calculated data, the latter quantifies the deviation between the estimated and (exactly) known model

$$
\begin{aligned}
D^{(d)} & =\sqrt{\frac{1}{P N} \sum_{p=1}^{P} \sum_{k=1}^{N}\left(\frac{d_{p k}^{(m)}-d_{p k}^{(c)}}{d_{p k}^{(m)}}\right)^{2}} \cdot 100(\%), \\
D^{(m)} & =\sqrt{\frac{1}{L M} \sum_{l=1}^{L} \sum_{i=1}^{M}\left(\frac{m_{l i}^{(t)}-m_{l i}^{(e)}}{m_{l i}^{(e)}}\right)^{2}} \cdot 100(\%),
\end{aligned}
$$

where $\boldsymbol{m}^{(e)}$ and $\boldsymbol{m}^{(t)}$ denote the estimated and target model, respectively ( $L$ is the number of layers, $M$ is the number of model parameters). The outputs of inversion program runs in Table 1 show highly accurate estimation results. We let the search run till 15,000 generations in both cases (Figure 1). The inversion procedures were stable and the data distance of the solutions based on Equation (7) was around 5.1\% (Figure 2(a)). The model distance for fitness function $F_{1}$ was $0.85 \%$, while that of $F_{2}$ was $0.62 \%$ (Figure 2(b)). It was concluded that the application of both fitness functions led to the same (global) optimum. In case of $F_{2}$ a faster convergence towards the optimum was found after iteration 1000, and a little higher retrieval accuracy was indicated as the relative improvement of model distance was $37 \%$ compared to the case of $F_{1}$.

After defining a proper fitness function, the upper and lower boundaries of model parameters and the control parameters of genetic operators must be specified (specifying search domain in Figure 1). The optimal values of series expansion coefficients $\boldsymbol{B}$ are estimated by the GA-based interval inversion method, which are substituted into Equation (4) to produce the vertical distributions of petrophysical parameters. The determination of layer-thicknesses cannot be accomplished with local inversion methods. They have to be a priori known before starting a local inversion procedure. In interval inversion only the lower and upper limits of thicknesses are required that means a non-automatic processing step. To automate this phase, we use cluster analysis for producing the estimates of layer-thicknesses that can be treated 
(a)

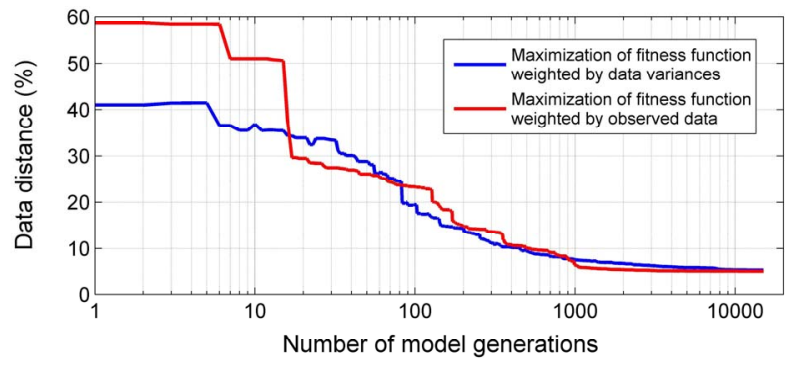

(b)

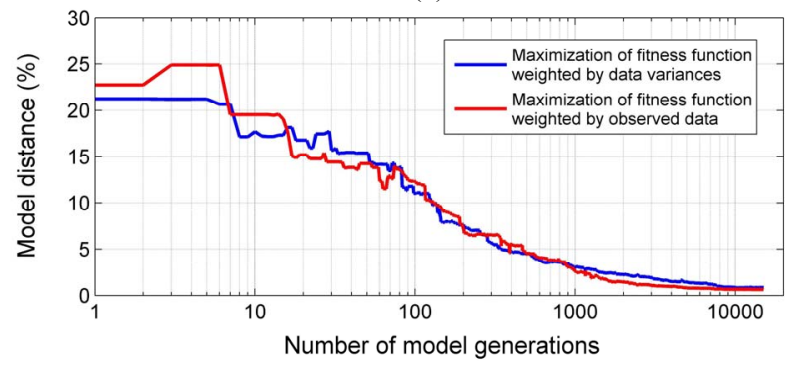

Figure 2. Convergence of interval inversion procedures.

either as constant or unknown in the interval inversion procedure.

\subsection{Hierarchical Cluster Analysis}

Clustering methods can be effectively used for the grouping of well-logging data in such a way that the $N$ dimensional objects specified by data sets measured from given depths are more similar than others observed from different depths. From the point of view of our method, it is of great importance that objects connected to the same cluster define approximately the same lithologic character, while other clusters represent dissimilar ones.

Agglomerative clustering methods build a hierarchy from the observed objects by progressively merging clusters. At the beginning, we have as many clusters as individual elements. In the first step, the closest points are coupled together to form a new cluster. In each following step, the distances between objects are re-calculated and the procedure is continued until all elements are grouped into one cluster. In this study, we used the matrix of Euclidean distances as a measure of dissimilarity between the pairs of observed objects. During the procedure the distances between the elements of the same group are minimized while they are maximized between the clusters at the same time. During the reconnection of clusters we follow the Ward's linkage criterion that minimizes the deviances of $\left(x_{i}\right.$-c), where $x_{i}$ is the $i$-th object and $\mathrm{c}$ is the centroid (average of elements) of the given cluster [22]. The result of cluster analysis is a dendrogram that shows the steps of clustering as it provides the hierarchy of clusters and the connections between them at different distances.
We use cluster analysis as a preliminary data processing step before inverse modeling (Figure 1). The input of clustering is the complete data set originated from the logged interval. By finding the similarities between raw data the objects are grouped into clusters. The log of clusters correlates well to the lithology variation along a borehole. The change in the group number of clusters appearing on the log gives the positions of layer boundaries, which can be read automatically by computer processing. The estimated layer-boundary coordinates as important a priori information for constructing the starting model serve as input for the interval inversion procedure.

\section{Hydrocarbon Field Application}

We tested the inversion method on a borehole geophysical data set measured from a Hungarian hydrocarbon well (Well No. 1). We used $G R, K, U, T H, D E N, N P H I$, $A T, R T \operatorname{logs}$ as input for the interpretation procedure. In the processed interval a sedimentary complex made up of four unconsolidated shaly-sand beds is found. The crossover effect between DEN and NPHI logs confirms the presence of gas in the porous and permeable formations. According to the workflow in Figure 1, at first a simultaneous cluster analysis of the 8 logs was performed. We specified three lithological categories: shale, shaly sand and sand. At this stage of interpretation, this resolution is enough for finding the layer-boundaries, because the relative volumes of rock-forming sand and shale can be estimated later in the inversion processing phase. For measuring the distance between the observed objects we used a standardized Euclidean distance, where each datum in the sum of squares is inversely weighted by the sample variance of that data type. We assigned leaf node numbers for each object in the original data set, where some leaf nodes correspond to multiple objects (Figure 3(b)). We created the hierarchical cluster tree of 30 objects by using the Ward's linkage algorithm (Figure 3(a)).

On the dendrogram 3 clusters can be discriminated at distance 15. The 3D crossplots of clustered well-logging data can be seen in Figures 4-6. The plots give useful information about some site-specific constants for calculating wellbore data in the straightforward modeling phase (well-logging data prediction in Figure 1) as values of physical properties of rock constituents (sand, shale) must be specified in probe response equations. For instance, the neutron porosity of sands $(15 \%)$ and shale $(26 \%)$ or the natural gamma-ray intensity of sand (40 API) and shale (110 API) can be estimated for the given hydrocarbon zone (Figure 5).

The log of the group numbers of clusters is useful to separate three types of formations (sand reservoir, shale, shaly-sand reservoir). The layer-boundaries are normally picked out at the places of inflection points on the $G R$ 

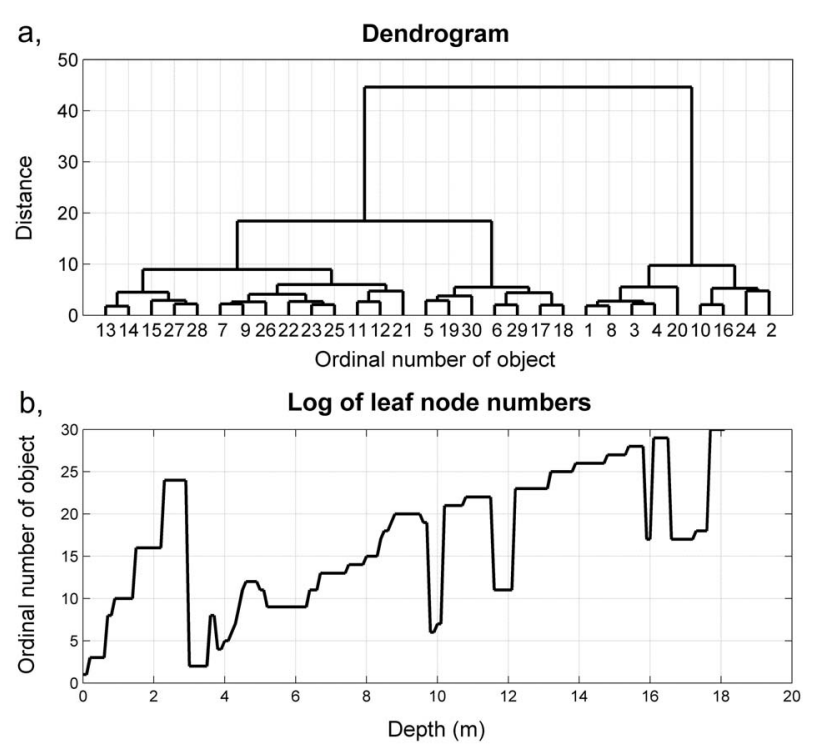

Figure 3. Result of cluster analysis in Well No. 1.

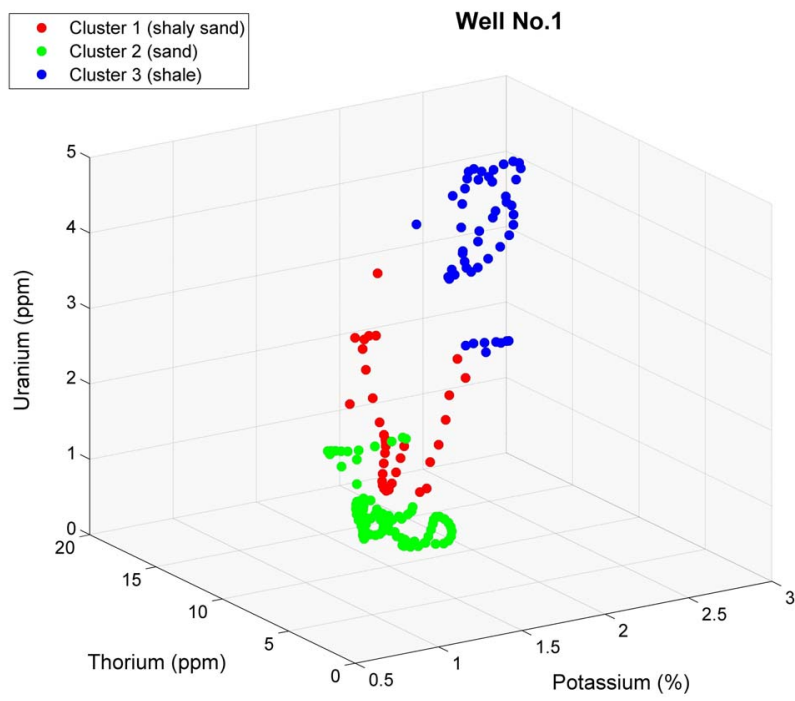

Figure 4. Clustered spectral gamma-ray intensity log data in Well No. 1.

curve. These depths are well indicated by abrupt changes in cluster log. In the given example, the layer-boundary coordinates were found at $4 \mathrm{~m}, 8.4 \mathrm{~m}, 9.6 \mathrm{~m}, 16 \mathrm{~m}$. We set these coordinates fixed, then petrophysical parameters were determined by interval inversion.

In the interval inversion phase, we applied a series expansion of model parameters ( $P O R, S X 0, S W, V S H, V S D)$ for a combination of homogeneous and inhomogeneous layers. Until the depth of $9.6 \mathrm{~m}$ the 3 layers can be treated as homogeneous ones. Below $10 \mathrm{~m}$ the 2 statistically located layers can be coupled as they belong to the same hydrocarbon reservoir. For the first 3 layers unit step functions, for the rest ones fourth-degree power functions were used as basis functions in Equation (4).

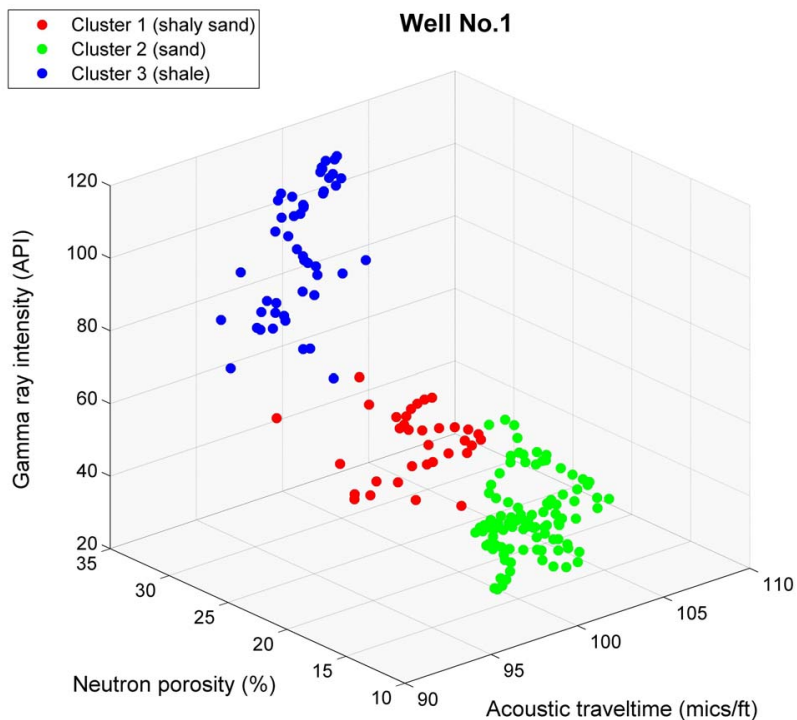

Figure 5. Clustered neutron, acoustic and natural gammaray intensity log data in Well No. 1.

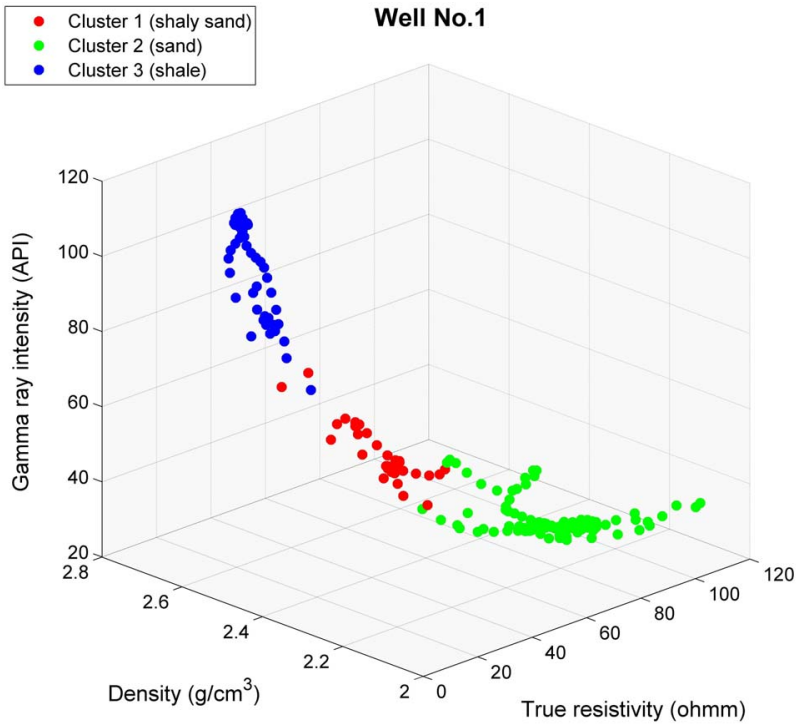

Figure 6. Clustered bulk density, resistivity and natural gamma-ray intensity log data in Well No. 1.

The standalone GA procedure is very time-consuming. For decreasing the CPU time of the inversion procedure we implemented a hybrid optimization technique that is based on the successive combination of global and linearized optimization methods [8]. We start the procedure with global optimization (GA) that performs a random search in the parameter space avoiding the local maxima of $F_{2}$ in Equation (6). Then, after some 200 generations, in the near vicinity of the absolute maximum, we change for a faster linear optimization method (Figure 7). We used the Damped Least Squares (DLSQ) method for the minimization of objective function $E^{*}=-F_{2}$ in the sec- 


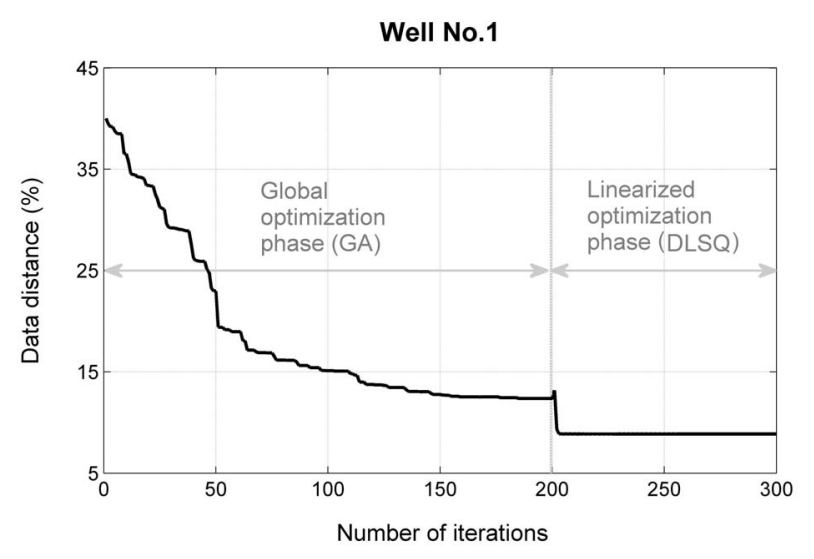

Figure 7. Convergence of GA + DLSQ interval inversion procedure in Well No. 1.

ond phase with applying proper damping factor to avoid big skips from the optimum [19]. This method both accelerates the rate of convergence of the inversion procedure and gives the estimation errors of model parameters as the Jacobi's matrix is calculated by Equation (3) at the end of the procedure.

The petrophysical parameters and their estimation errors are listed in Table 2. In the $4^{\text {th }}$ layer, the depth coordinates were properly transformed into the range of 0 and 1 for the polynomial approximation, where $C_{0}$ denotes the coefficient of the $0^{\text {th }}$ power exponent of depth coordinate as independent variable. The inversion results are highly accurate according to the values of estimation errors. This is because of the high overdetermination of the inverse problem. For further increasing the overdetermination, parameter $V S D$ in the $4^{\text {th }}$ layer was calculated by the physical constraint $V S D=1-P O R-V S H$. The measured logs and inversion results are in Figure 8. The log of the group numbers of clusters is represented in track 6, while the inversion estimates are in tracks 7 8 , where the relative volume of water, movable and ireducible hydrocarbon, pore space, shale, sand can be analyzed visually. In the $4^{\text {th }}$ layer, gas is accumulated at the top of the reservoir, while the denser water is situated underneath. The amount of shale is increasing with depth in the lowest formation.

\section{Conclusions}

The ever-increasing claim of oil industry lain to highly reliable petrophysical information requires advanced data processing techniques. In the paper, a quick automated inversion method was shown for the interpretation of borehole geophysical data. The cluster analysis assisted joint inversion method is highly accurate (assured by great amount of overdetermination), automatic (automated layer boundary and petrophysical model estimation), robust and adaptive (evolutionary algorithm phase)
Table 2. GA interval inversion results in Well No. 1.

\begin{tabular}{|c|c|c|c|c|c|}
\hline \multirow{2}{*}{ Layer } & \multicolumn{5}{|c|}{ Petrophysical parameters as inversion unknowns } \\
\hline & POR & $S X 0$ & $S W$ & VSH & $V S D$ \\
\hline 1 & $\begin{array}{c}0.10 \\
( \pm 0.002)^{*}\end{array}$ & $\begin{array}{c}0.99 \\
( \pm 0.02)\end{array}$ & $\begin{array}{c}0.99 \\
( \pm 0.02)\end{array}$ & $\begin{array}{c}0.58 \\
( \pm 0.003)\end{array}$ & $\begin{array}{c}0.32 \\
( \pm 0.003)\end{array}$ \\
\hline 2 & $\begin{array}{c}0.27 \\
( \pm 0.002)\end{array}$ & $\begin{array}{c}0.75 \\
( \pm 0.004)\end{array}$ & $\begin{array}{c}0.23 \\
( \pm 0.002)\end{array}$ & $\begin{array}{c}0.07 \\
( \pm 0.001)\end{array}$ & $\begin{array}{c}0.66 \\
( \pm 0.002)\end{array}$ \\
\hline 3 & $\begin{array}{c}0.17 \\
( \pm 0.003)\end{array}$ & $\begin{array}{c}0.99 \\
( \pm 0.02)\end{array}$ & $\begin{array}{c}0.99 \\
( \pm 0.02)\end{array}$ & $\begin{array}{c}0.34 \\
( \pm 0.003)\end{array}$ & $\begin{array}{c}0.49 \\
( \pm 0.003)\end{array}$ \\
\hline 4 & $\begin{array}{c}\mathrm{C}_{0}=0.277 \\
( \pm 0.002) \\
\mathrm{C}_{1}=0.026 \\
( \pm 0.006) \\
\mathrm{C}_{2}=-0.001 \\
( \pm 0.000) \\
\mathrm{C}_{3}=-0.007 \\
( \pm 0.000) \\
\mathrm{C}_{4}=-0.1734 \\
( \pm 0.007)\end{array}$ & $\begin{array}{c}\mathrm{C}_{0}=0.679 \\
( \pm 0.01) \\
\mathrm{C}_{1}=0.592 \\
( \pm 0.108) \\
\mathrm{C}_{2}=-0.776 \\
( \pm 0.406) \\
\mathrm{C}_{3}=-0.740 \\
( \pm 0.633) \\
\mathrm{C}_{4}=1.350 \\
( \pm 0.346)\end{array}$ & $\begin{array}{c}\mathrm{C}_{0}=0.253 \\
( \pm 0.002) \\
\mathrm{C}_{1}=-0.994 \\
( \pm 0.025) \\
\mathrm{C}_{2}=4.027 \\
( \pm 0.111) \\
\mathrm{C}_{3}=-6.275 \\
( \pm 0.201) \\
\mathrm{C}_{4}=3.696 \\
( \pm 0.123)\end{array}$ & $\begin{array}{c}\mathrm{C}_{0}=0.069 \\
( \pm 0.002) \\
\mathrm{C}_{1}=-0.524 \\
( \pm 0.0346) \\
\mathrm{C}_{2}=1.860 \\
( \pm 0.149) \\
\mathrm{C}_{3}=-1.852 \\
( \pm 0.237) \\
\mathrm{C}_{4}=0.722 \\
( \pm 0.123)\end{array}$ & 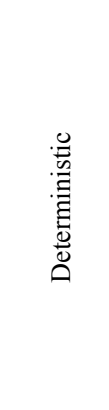 \\
\hline
\end{tabular}

*Estimation error of the model parameter in fractional unit.

and fast (hybrid optimization technique; simultaneous inversion processing of data from the logged interval instead of one point).

The inversion method is not fully automatic, where the supervision of the $\log$ analyst cannot be discarded. To achieve a good and unique solution, the prior geological and geophysical information must be built-in by the user properly (chose of site specific constants and response equations in different hydrocarbon zones). Moreover, in case of the global optimization phase (GA), some experience is needed to set the combination and control parameters of genetic operators and to decide when it is possible to switch over to linear optimization. As a speciality of the interval inversion method, the basis functions of series expansion can be chosen arbitrarily. The optimal set of basis functions to be in use depends on the variation of lithology and pore fluids along a borehole. A trade-off must be taken between the vertical resolution (number of unknowns) and stability (uniqueness) of the inversion procedure as they are inversely proportional. Usually, this is the most important question from the point of view of constructing an inversion method. To increase the overdetermination of the inverse problem, it is important to search for such parameters that can be fixed during the inversion procedure. The cluster analysis-based interval inversion method helps to find lithological similarities in the data set, which leads to constrain the inversion process with reliable site constants and layerthicknesses. This can reduce the uncertainty and ambiguity of inversion estimates. In the future, we are planning 


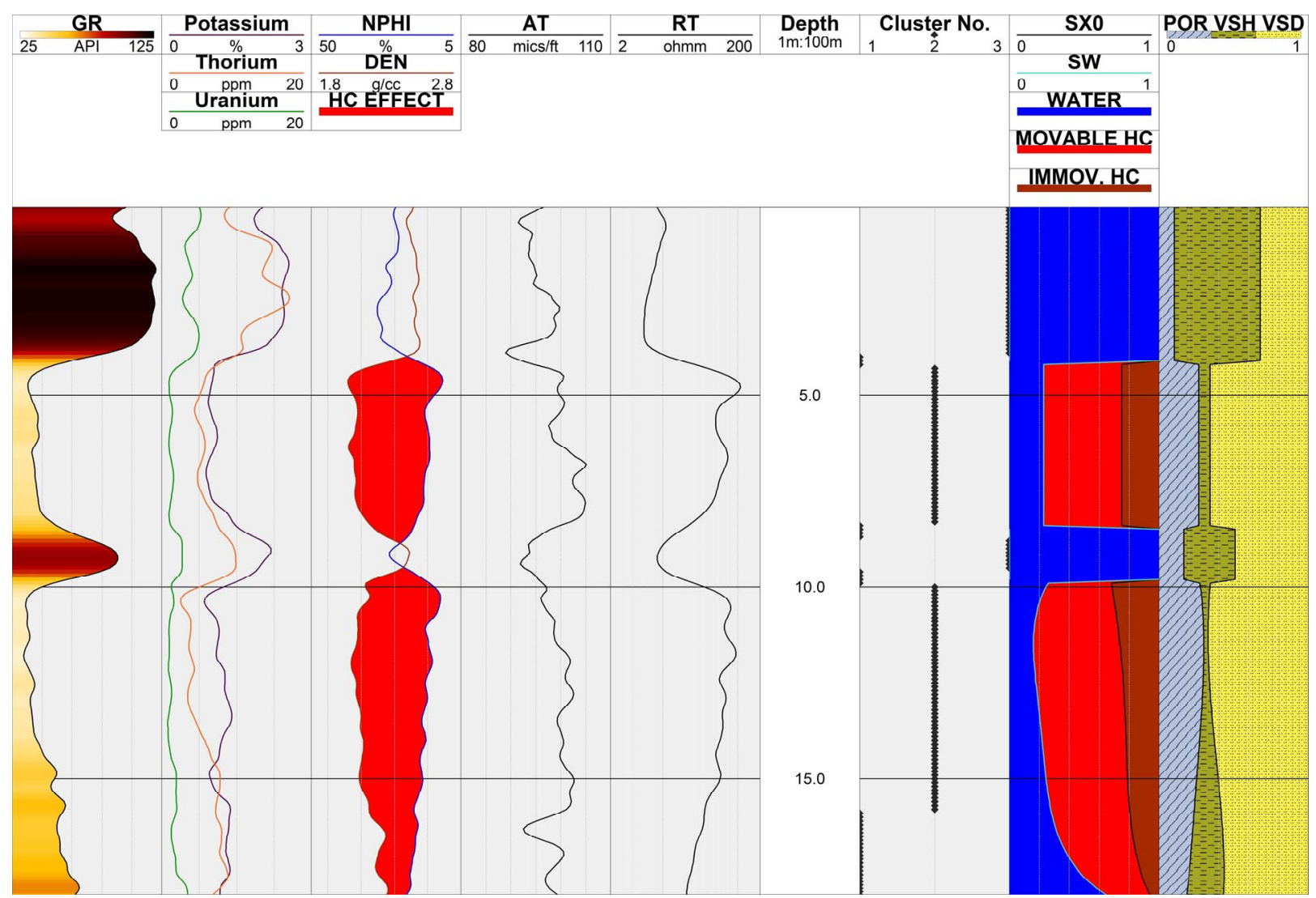

Figure 8. Processed well logs and result of cluster analysis assisted interval inversion procedure.

to find technical solutions to reach even better spatial resolution of petrophysical properties with preserving stability by using orthogonal polynomial series expansion along the entire logging interval and further develop the multiwell applications of the presented inversion method.

\section{Acknowledgements}

The described work was carried out as part of the TÁMOP-4.2.2/B-10/1-2010-0008 project in the framework of the New Hungary Development Plan. The realization of this project is supported by the European Union, co-financed by the European Social Fund. N. P. Sz. as the leading researcher of project no. PD 109408 thanks to the support of the Hungarian Scientific Research Fund. M. D. as the leading researcher of project no. K 109441 thanks to the support of the Hungarian Scientific Research Fund. N. P. Sz. thanks to the support of the János Bolyai Research Fellowship of the Hungarian Academy of Sciences. We thank the long-term co-operation to the Hungarian Oil and Gas Company.

\section{REFERENCES}

[1] D. V. Ellis and J. M. Singer, "Well Logging for Earth
Scientists," 2nd Edition, Springer, Dordrecht, 2007.

[2] M. Alberty and K. Hashmy, "Application of ULTRA to log Analysis," SPWLA Symposium Transactions, 1984, pp. 1-17.

[3] S. M. Ball, D. M. Chace and W. H. Fertl, "The Well Data System (WDS): An Advanced Formation Evaluation Concept in a Microcomputer Environment," Proceedings of SPE Eastern Regional Meeting, Pittsburgh, 1987, pp. 61-85. http://dx.doi.org/10.2118/17034-MS

[4] C. Mayer, "GLOBAL, a New Approach to ComputerProcessed Log Interpretation," Proceedings of 55th SPE Annual Technical Conference and Exhibition, 1980, pp. 1-14. http://dx.doi.org/10.2118/9341-MS

[5] M. Dobróka and N. P. Szabó, "Interval Inversion of Well-Logging Data for Automatic Determination of Formation Boundaries by Using a Float-Encoded Genetic Algorithm," Journal of Petroleum Science and Engineering, Vol. 86-87, 2012, pp. 144-152. http://dx.doi.org/10.1016/j.petrol.2012.03.028

[6] M. Dobróka, N. P. Szabó and E. Turai, "Interval Inversion of Borehole Data for Petrophysical Characterization of Complex Reservoirs," Acta Geodaetica et Geophysica Hungarica, Vol. 47, No. 2, 2012, pp. 172-184. http://dx.doi.org/10.1556/AGeod.47.2012.2.6

[7] M. Dobróka, P. N. Szabó, E. Cardarelli and P. Vass, "2D Inversion of Borehole Logging Data for Simultaneous Determination of Rock Interfaces and Petrophysical Parameters," Acta Geodaetica et Geophysica Hungarica, 
Vol. 44, No. 4, 2009, pp. 459-479. http://dx.doi.org/10.1556/AGeod.44.2009.4.7

[8] M. Dobróka and P. N. Szabó, "Combined Global/Linear Inversion of Well-Logging Data in Layer-Wise Homogeneous and Inhomogeneous Media," Acta Geodaetica et Geophysica Hungarica, Vol. 40, No. 2, 2005, pp. 203214. http://dx.doi.org/10.1556/AGeod.40.2005.2.7

[9] N. P. Szabó and M. Dobróka, "Float-Encoded Genetic Algorithm Used for the Inversion Processing of WellLogging Data," In: A. Michalski, Ed., Global Optimization: Theory, Developments and Applications, Mathematics Research Developments, Computational Mathematics and Analysis Series, Nova Science Publishers Inc., Hauppauge NY, 2013, pp. 79-104.

[10] W. B. Hempkins, "Multivariate Statistical Analysis in Formation Evaluation," SPE California Regional Meeting, 1978, pp. 1-20. http://dx.doi.org/10.2118/7144-MS

[11] G. N. Lance and W. T. Williams, "A General Theory of Classificatory Sorting Strategies 1. Hierarchical Systems," The Computer Journal, Vol. 9, No. 4, 1967, pp. 373-380. http://dx.doi.org/10.1093/comjnl/9.4.373

[12] J. Kovács, P. Tanos, J. Korponai, I. K. Székely, K. Gondár, K. Gondár-Sőregi and I. G. Hatvani, "Analysis of Water Quality Data for Scientists," In: K. Voudouris and D. Voutsa, Eds., Water Quality Monitoring and Assessment, InTech Open Access Publisher, Rijeka, 2012, pp. 65-94.

[13] M. Kazmierczuk and J. Jarzyna, "Improvement of Lithology and Saturation Determined from Well Logging Using Statistical Methods," Acta Geophysica, Vol. 54, No. 4, 2006, pp. 378-398. http://dx.doi.org/10.2478/s11600-006-0030-y
[14] V. Tavakoli and A. Amini, "Application of Multivariate Cluster Analysis in Logfacies Determination and Reservoir Zonation, Case Study of Marun Field, South of Iran," Journal of Science University of Teheran, Vol. 32, No. 2, 2006, pp. 69-75.

[15] H. H. Perez, A. Datta-Gupta and S. Mishra, "The Role of Electrofacies, Lithofacies, and Hydraulic Flow Units in Permeability Predictions from Well Logs: A Comparative Analysis Using Classification Trees," SPE Annual Technical Conference and Exhibition, Denver, 2003, pp. 1-11. http://dx.doi.org/10.2118/84301-MS

[16] G. Asquith and D. Krygowski, "Basic Well Log Analysis," 2nd Edition, AAPG, Tulsa, 2004.

[17] M. H. Rider," The Geological Interpretation of Well Logs," 2nd Edition, Rider-French Consulting Ltd., Sutherland, 2002.

[18] Á. Gyulai, M. K. Baracza and É. E. Tolnai, "The Application of Joint Inversion in Geophysical Exploration," International Journal of Geosciences, Vol. 4, No. 2, 2013, pp. 283-289. http://dx.doi.org/10.4236/ijg.2013.42026

[19] W. Menke, "Geophysical Data Analysis: Discrete Inverse Theory," Academic Press Inc., New York, 1984.

[20] J. H. Holland, "Adaptation in Natural and Artificial Systems," University of Michigan Press, Ann Arbor, 1975.

[21] Z. Michalewicz, "Genetic Algorithms Plus Data Structures Equals Evolution Programs," Springer-Verlag Inc., New York, 1992.

[22] J. H. Ward, "Hierarchical Grouping to Optimize an Objective Function," Journal of the American Statistical Association, Vol. 58, No. 301, 1963, pp. 236-244. http://dx.doi.org/10.1080/01621459.1963.10500845 